\title{
Civilisations
}

Revue internationale d'anthropologie et de sciences

humaines

$67 \mid 2018$

À l'écoute des transnationalisations religieuses

\section{Composer avec les orishas. Acteurs et actrices de la religiosité du candombe afro-uruguayen}

Composing with orishas. Actors of religiosity in the Afro-Uruguayan candombe

\section{Clara Biermann}

\section{OpenEdition}

Journals

Édition électronique

URL : https://journals.openedition.org/civilisations/5069

DOI : 10.4000/civilisations.5069

ISSN : 2032-0442

\section{Éditeur}

Institut de sociologie de l'Université Libre de Bruxelles

\section{Édition imprimée}

Date de publication : 12 août 2018

Pagination : 129-149

ISBN : 978-2-9602017-2-7

ISSN : 0009-8140

Référence électronique

Clara Biermann, «Composer avec les orishas. Acteurs et actrices de la religiosité du candombe afrouruquayen », Civilisations [En ligne], 67 | 2018, mis en ligne le 01 janvier 2022, consulté le 08 janvier 2022. URL : http://journals.openedition.org/civilisations/5069 ; DOI : https://doi.org/10.4000/ civilisations.5069 


\section{Composer avec les orishas}

\section{Acteurs et actrices de la religiosité du candombe afro-uruguayen}

\section{Clara BIERMANN}

Résumé : A partir d'une analyse des trajectoires et des compositions musicales de Pedro Ferreira (1910-1980) et Chabela Ramírez (née en 1958), cet article explore la manière dont ces deux figures ont joué et jouent le rôle de « passeurs » de mythes associés aux orishas, les divinités d'origine yoruba des religions afro-cubaines et afro-brésiliennes, dans le répertoire chanté du candombe afro-uruguayen. En resituant les sources et les enjeux de la création de leurs chansons, deux périodes seront ainsi mises en relation. La première est celle de l'appropriation des musiques afro-cubaines inspirées des pratiques religieuses, à partir des années 1950, dans la phase de «tropicalisation » du candombe. La deuxième, à partir de la fin des années 1990, concerne la resémantisation religieuse du candombe par le biais d'instruments, de rythmiques, de contenus poétiques sur les orishas, dans un contexte d'expansion des religions afro-brésiliennes en Uruguay, initiée depuis les années 1950. En composant avec les orishas, ces musiciens ont conféré au candombe une sonorité afro associée à une sacralité, dans un mouvement de construction d'afrodescendance en Uruguay, qui, tout en puisant dans une culture religieuse afro transnationale, soutient la création d'une mémoire culturelle spécifique dans l'espace national uruguayen.

Mots-clés : candombe afro-uruguayen, appropriation, mémoire(s), afrodescendance, Uruguay

Abstract: Based on an analysis of the trajectories and musical compositions of Pedro Ferreira (1910-1980) and Chabela Ramírez (born 1958), this article explores how these two musicians have played and continue to play a role in passing on the myths associated with orishas - the Yoruba deities of Afro-Cuban and Afro-Brazilian religions - in the repertoire of songs of the the Afro-Uruguayan candombe. Putting into context the sources and the issues linked to the creation of their songs, two periods will be compared. The first is that of the appropriation from the 1950 s onwards of Afro-Cuban music inspired by religious practices during the phase of the "tropicalization" of candombe. The second period concerns the religious re-signifying of candombe through instruments, rhythms, and poetic content regarding orishas which begun at the end of the 1990s in a context of expansion of Afro-Brazilian religions in Uruguay (which started in the 1950s). By composing with the orishas, these musicians have conferred on the candombe an African sound associated with a sacred dimension in a movement of construction of Afrodescendancy in Uruguay, which, while drawing on an transnational African religious culture, supports the creation of a specific cultural heritage in the Uruguayan national space.

Keywords: Afro-Uruguayan candombe, appropriation, heritage, afrodescendancy, Uruguay 


\section{Introduction}

« J'ai rencontré une femme un jour qui m'a dit : “Vous les Noirs, vous ne savez pas exactement ce qui vous unit. Vous les Noirs, vous ne savez rien de votre religiosité". Je lui ai répondu que c'était vrai et je lui ai demandé si elle allait me l'apprendre. Elle me ramène alors un livre. Et là, je n'en reviens pas de ce que je découvre. La force de la nature, c'est-à-dire les orishas, je n'avais rien vu de tel dans ma vie. A peine avais-je entendu dans quelques chansons de Pedro Ferreira parler des orishas comme Shangó et lemanjá. C'était un monde nouveau, je ne savais pas ce que c'était l'umbanda, le batuque, rien. Mais je voulais connaître cet héritage que je portais. (...) Et quand j'ai fait la connaissance des orishas, je suis tombée amoureuse, éperdument amoureuse. Pas seulement des images auxquelles je pouvais m'identifier du point de vue de la couleur de peau - alors que Dieu est blanc avec les yeux bleus - mais aussi des qualités de chacun d'entre eux $»^{1}$.

C'est ainsi que Chabela Ramírez, chanteuse de candombe et militante afro-uruguayenne, décrit sa rencontre avec les orishas, les divinités d'origine yoruba qui font l'objet d'un culte dans les religions afro-brésiliennes et afro-cubaines ${ }^{2}$. Malgré sa ressemblance avec son quasi-homonyme « candomble », la religion afro-brésilienne bien connue des études afro-américanistes françaises (Bastide [1958] 2000 ; Capone 1999), le candombe afro-uruguayen est un genre musico-chorégraphique polymorphe qui se pratique aujourd'hui sous différentes formes dans des contextes festifs et carnavalesques ${ }^{3}$. Si une dimension religieuse existait certainement dans ce qu'on appelait «candombe » au début du $19^{\mathrm{e}}$ siècle, c'est-à-dire les pratiques musicales et dansées des populations africaines réduites en esclavage à Montevideo (Ayestarán 1953), le processus de spectacularisation qu'elles ont connu à partir de la deuxième moitié du $19^{\mathrm{e}}$ siècle, dans

1 Propos recueillis lors de la présentation de ma thèse à la Casa de la Cultura Afrouruguaya, à Montevideo, le 4 mars 2016.

2 Que le lecteur ne se formalise pas, il trouvera dans ce texte le terme orisha parfois écrit orixá, comme au Brésil. Par ailleurs, en dehors des archives citées ou du nom des initiés, j'utilise pour le nom de chaque orisha, l'orthographe la plus fréquemment utilisée en Uruguay.

3 Le candombe est joué pendant le Carnaval de Montevideo, un concours sur scène d'une quarantaine de jours qui comprend cinq catégories, dont l'une, les Sociétés de Noirs et de Lubolos, présente des spectacles de théâtre chanté (le terme « lubolo » désigne les Blancs qui se peignaient le visage en noir et imitaient les pratiques musicales et dansées des Noirs au $19^{\mathrm{e}}$ siècle). Le candombe est également joué en llamada (appel), c'est-à-dire en cortège par des groupes d'une quarantaine de tambours et d'autant de danseurs et de danseuses. Cette pratique donne lieu à un concours annuel, le premier week-end du mois de février à Montevideo, appelé le Desfile de Llamadas. Par ailleurs, il existe plusieurs genres de candombe chanté qui se sont développés depuis les années 1920 comme le candombe-tango, le candombe-rock ou le candombe-jazz (Ferreira 1997 ; Aharonián 2010 ; Picún 2016). Le dénominateur commun à toutes ces formes musicales est la présence des tambores, des membranophones en forme de baril à une peau, percutés avec main et baguette, qui jouent une polyrythmie construite sur l'entrecroisement du jeu de trois tambours, appelés du plus aigu au plus grave : le chico, le repique et le piano. 
un contexte de sécularisation et de «blanchiment » de la société uruguayenne (Ferreira 2003b ; Andrews 2011), en a effacé les traces explicites ${ }^{4}$.

Après plusieurs décennies de transformations et d'adaptations tant sociales que musicales, le candombe a fait l'objet, à partir de la fin de la dictature militaire (19731985), d'une appropriation d'une ampleur sans précédent. Les Uruguayens de toutes origines, les habitants des villes de l'intérieur du pays et les migrants uruguayens en Europe, aux Etats-Unis et en Australie se sont mis à jouer des tambours en cortège. Cette pratique, appelée llamada (appel) et qui renvoie au système d'appel-réponse qui caractérise cette polyrythmie, était jusqu'alors circonscrite à trois quartiers du centreville de Montevideo à forte densité de population afro-uruguayenne: les quartiers Sur, Palermo et Cordón. Cet engouement populaire, conjugué à l'usage du candombe comme instrument de musical construction of place (Stockes 1994) par les organisations noires et à l'intérêt de la gauche uruguayenne à s'inscrire dans le multiculturalisme et la défense des droits des populations indigènes et afrodescendantes en Amérique latine prônée par les instances internationales (Cunin 2006), ont conduit à la patrimonialisation du candombe, déclaré Patrimoine National en 2006 et ajouté à la liste du Patrimoine Culturel Immatériel de l'UNESCO en 2009.

Dans ce contexte aux enjeux multiscalaires, mon travail de recherche s'est intéressé aux positionnements esthétiques des musiciens afro-uruguayens et des musiciennes et danseuses afro-uruguayennes, revendiquant une propriété culturelle sur le candombe par sa « réafricanisation» (Biermann 2011, 2015), notion que je définis ici comme un processus de sémantisation du candombe par des éléments sonores, gestuels et poétiques associés à l'Afrique, qui s'inscrit dans une volonté de retour aux origines. Or ces éléments utilisés par les artistes afro-uruguayens sont principalement issus des religions afro-brésiliennes, lesquelles se sont implantées en Uruguay à partir de la fin des années 1950. L'Uruguay a été en effet le premier terrain du phénomène de transnationalisation ${ }^{5}$ des «religions des orishas » (Argyriadis \& Capone 2011),

4 Ceci explique certainement que le candombe n'ait jamais retenu l'attention de Roger Bastide. Cette pratique carnavalesque d'un pays faisant partie de «l'Amérique blanche » ([1967] 1996:19) n'est pas assez religieuse ni assez africaine pour lui, ni ensuite pour un champ de recherche qui se construit dans un rapport ambigu à la question des survivances africaines dans le Nouveau Monde (Capone 2005a).

5 La transnationalisation (religieuse) désigne ici la relocalisation de pratiques religieuses, de croyances et de rituels au-delà de leur cadre national d'origine, qui s'accompagne de nouveaux imaginaires et récits identitaires dans les territoires où ces religions s'implantent. Pour une discussion approfondie autour de ce terme, voir Capone 2004. 
plus particulièrement l'umbanda et le batuque ${ }^{6}$, que l'on désigne localement comme les religiones afro (« religions afros »). Si les modalités d'implantation de l'umbanda et du batuque dans le champ religieux uruguayen ont été retracées (Pi Hugarte 1998 ; Frigerio 1998, 2011), mes enquêtes ethnographiques, menées depuis 2007 à Montevideo, invitent à considérer le rôle du candombe, en tant qu'emblème de la culture afro-uruguayenne, dans cette incorporation des religiones afro à la culture nationale.

A partir d'une analyse des trajectoires et des productions musicales de deux figures du candombe, le chanteur et compositeur Pedro Ferreira (1910-1980) et la chanteuse et militante Chabela Ramírez (1958), mon propos est de comprendre comment ces deux artistes ont joué et jouent le rôle de "passeurs » de mythes et de sons associés aux religiones afro, tout en resituant les enjeux esthétiques et politiques qui traversent leurs créations musicales. Cette analyse s'appuie sur un travail d'archives et le recueil de sources orales pour Pedro Ferreira, et sur les données de l'enquête ethnographique que je mène avec Chabela Ramírez depuis plus d'une dizaine d'années. Mon travail de recherche m'a permis d'identifier deux phases qui caractérisent les modalités d'articulation entre le candombe, les religiones afro et la construction d'afrodescendance en Uruguay, la première, datant des années 1950, dans un contexte de circulations musicales et culturelles à l'échelle globale et de «tropicalisation » du candombe (Aharonían 2010 ; Ferreira 1997), et la seconde, depuis les années 1990, dans le cadre d'un militantisme politique et culturel articulé à un phénomène de transnationalisation religieuse.

En ce sens, cet article entre en résonance avec les recherches qui se sont intéressées, en d'autres lieux, à la relation entre africanité et religion (pour les Etats-Unis, Capone 2005b; Guedj 2011), et celles qui ont montré comment cette relation se construit par l'appropriation d'éléments religieux dans des pratiques musico-chorégraphiques carnavalesques, comme à Salvador de Bahia par exemple (Agier 2000). De même, mon propos s'inscrit dans la continuité des travaux qui envisagent la manière dont la sacralité de la musique religieuse peut être adaptée, appropriée, et participer à la fabrication d'appartenances multiples et de mémoire(s) culturelle(s) (Sullivan 1997).

6 Le batuque est une religion afro-brésilienne originaire de l'Etat de Rio Grande do Sul, région frontalière avec l'Uruguay, qui présente de fortes similitudes avec le candomblé originaire de Salvador de Bahia. Le candomblé, l'umbanda et le batuque ont en commun, malgré certaines différences cosmogoniques et liturgiques, de reconnaître l'existence d'un dieu suprême, appelé Olorum, à la volonté duquel sont soumis l'ensemble des êtres vivants et toutes les entités spirituelles, dont les orishas, qui servent d'intermédiaires entre Olorum et les hommes (Capone 1999 : 337 ; Halloy 2005 : 141). Les orishas, divinités du panthéon yoruba, sont des incarnations humanisées d'éléments de la nature, associées à des objets, couleurs, gestes et traits de caractères que l'on retrouve dans les mythes dont ils sont les protagonistes (Verger [1954] 1995). Au cours des cérémonies, ces divinités sont appelées par des chants spécifiques et s'incorporent à leurs « enfants » ou « descendent » sur eux. En effet, par l'initiation, chaque individu s'inscrit dans une relation de filiation vis-à-vis d'un orisha, dont il sera considéré comme le « fils » ou la « fille » et avec qui il entretient une relation à la fois de protection et d'obligation. Sur les spécificités du batuque, voir également Frigerio, <http://www.ird.fr/relitrans/spip.php?article319> 


\section{Appropriations afro-cubaines dans le candombe}

Montevideo est une ville portuaire, pôle des flux commerciaux et culturels de l'Atlantique sud reliant La Havane, Rio de Janeiro, Buenos Aires, l'Afrique et l'Europe. Dans les dernières décennies du $19^{\mathrm{e}}$ siècle, le Carnaval de Montevideo, dont les traces remontent au premier temps de la colonie, se transforme en un carnaval de spectacles sous l'impulsion des autorités de la ville qui encouragent la professionnalisation des ensembles qui y participent (Alfaro 1991). Ce sont les spectacles des Sociétés de Noirs et de Lubolos qui sont les plus appréciés du public. Composés d'Afro-Uruguayens, de criollos $^{7}$ et de migrants européens qui s'installent dans la capitale, ces troupes présentent des tableaux dansés accompagnés d'hymnes, de tangos, de candombes et de valses.

Dans les années 1920, de l'autre côté de l'Atlantique, les musiques et danses noires des trois Amériques connaissent un immense succès, notamment à Paris (Décoret-Ahiha 2004 ; Jacotot 2008). «Habilitées et installées mondialement par le goût parisien » et transmettant de ce fait « un message de sophistication, de cosmopolitisme et de raffinement urbain » dont le candombe ne bénéficiait pas localement (Andrews 2011 : 108), ces musiques afro-américaines, diffusées via l'industrie discographique, les ondes radiophoniques et les tournées des musiciens (Ferreira 2003a: 144), vont entrer au répertoire des Sociétés de Noirs et de Lubolos. Dans les années 1920, on écoute donc à Montevideo, comme me le raconte l'historien afro-uruguayen Tomás Olivera Chirimini, d'un côté de « la música típica (musique typique), c'est-à-dire tango, milonga et valse, et de l'autre on écoute à la radio la musique brésilienne : le maxixe, le samba, le choro et la musique cubaine, comme le Trio Matamoros $^{8}$, et puis aussi du jazz bien sûr $»^{9}$.

Deux décennies plus tard, le marché culturel international est caractérisé par l'omniprésence d'images et de sons venus des Caraïbes (Ávila Domínguez et al. 2011). Le $\operatorname{son}^{10}$, érigé en musique nationale sous l'impulsion du mouvement afrocubaniste (Moore 2002; Argyriadis 2005), mais aussi la rumba et la conga connaissent un rayonnement international par le biais des majors américaines comme RCA Victor et Columbia Records, qui inondent le marché avec ce qui est devenu l'un des meilleurs produits d'exportation de l'île (Leymarie 1997). Los Lecuona Cuban Boys ${ }^{11}$, La Sonora Matancera et sa charismatique chanteuse Celia Cruz ou encore l'orchestre féminin La Orchesta Anacoana, emblématiques de l'âge d'or du son cubain, font des tournées qui

7 J'utilise ici le terme criollo (créole) pour me référer aux descendants de colons nés sur le sol uruguayen, comme Carmen Bernand, sans le traduire, pour ne pas lui donner une connotation caribéenne (2013 : 193).

8 Le Trio Matamoros, formé en 1925, est un des groupes emblématiques de la trova cubaine, qui a fait des tournées en Europe et dans toute l'Amérique latine. Voir Leymarie 1997.

9 Extrait d'un entretien daté du 17 février 2012.

10 Le son, genre musical cubain, sera écrit en italique, à la différence des autres genres musicaux, pour ne pas le confondre avec le mot «son » en français.

11 Los Lecuona Cuban Boys, formés par le pianiste Ernesto Lecuona en 1933, sillonnent d'abord l'Europe, puis, à partir des années 1940, l'Amérique latine (Leymarie 1997 : 85). Ils deviennent des légendes locales en Uruguay, en écrivant une chanson en l'honneur du pays, La Conga del Carnaval del Uruguay, qui deviendra, d'après Tomás Olivera Chirimini, aussi connue que l'hymne national. 
passent par Montevideo. L'esthétique de ces «versions d'exportation » des orchestres cubains (Aharonián 2010 : 148) influence un certain Pedro Rafael Tabares, connu sous le nom de Pedro Ferreira (photo 1), célébré comme celui qui a su « faire une synthèse de ces mélodies [cubaines] avec notre rythme [de candombe]» et dont les pièces sont devenues des standards (Olivera Chirimini \& Varese 2000 : 73).

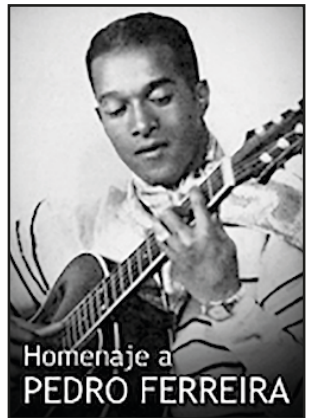

Photo 1. Affiche du concert d'Hommage à Pedro Ferreira, Montevideo : 3 décembre 2014, Jour National du Candombe

() Portal Candombe («www.candombe.com.uy»)

Pedro Ferreira est considéré comme le chanteur-compositeur le plus important du candombe de carnaval, mais peu connaissent son histoire aussi bien que l'historien Tomás Olivera Chirimini. Pendant mon séjour à Montevideo en 2012, je le rencontre dans son ancienne maison de famille devenue le local de son association culturelle, «Africanía », dans le quartier Palermo, qui renferme des trésors d'archives qu'il a collectées et garde précieusement. Dans le salon siègent un vieux piano désaccordé et des tambours de candombe. Sur le mur, des panneaux sont couverts de photographies et de coupures de journaux à la gloire du quartier et de ses figures légendaires. Parmi elles, des photos de Pedro Ferreira, que Tomás appelle affectueusement Pedrito.

Pedro naît en 1910. Par conséquent, de ses dix ans jusqu'à son adolescence, il s'imprègne à la fois de la musique brésilienne, qui était ce qui arrivait ici au Carnaval, et de la musique cubaine. Il est resté charmé par le son de tous ces orchestres, le Trío Matamoros, Los Lecuona Cuban Boys. Lui qui était d'une origine très modeste travaillait depuis tout jeune comme vendeur de journaux dans un coin de rue, tout près d'ici dans le quartier [Palermo]. Il s'avère qu'il avait un grand sens de la musique, alors il décide, vers l'âge de vingt ans, de tenter sa chance à Buenos Aires, où il travaille encore comme vendeur de journaux et cireur de chaussures. Et là-bas, il rencontre un groupe de musiciens cubains qui lui font connaître d'un peu plus près leur musique et il en sort complètement fasciné ${ }^{12}$.

La passion de Pedro Ferreira pour la musique afro-cubaine est en effet relatée par tous ceux qui l'ont connu et fait partie intégrante de sa légende. En rentrant à Montevideo, en 1944, après son expérience musicale avec le Trío Tropical à Buenos Aires, il intègre en tant que chanteur un groupe appelé Cubanacán et fait également ses débuts dans les Sociétés de Negros y Lubolos au Carnaval de Montevideo. C'est à partir de 1954 qu'il devient une figure du candombe de carnaval, en dirigeant le groupe Fantasía Negra du quartier Palermo, qui va gagner pendant cinq années consécutives le premier prix dans la catégorie Société de Noirs et de Lubolos. En 1957, il forme à nouveau un groupe qu'il dirige cette fois sous le nom de Pedro y su Cubanacán. Alexis Buenseñor, jeune 
trompettiste à l'époque, aujourd'hui directeur d'AGADU, l'Association Générale des Auteurs de l'Uruguay ${ }^{13}$, se rappelle quand Pedro Ferreira jouait avec la Cubanacán :

Je me souviens d'un thème, un tube qui rendait les gens fous. Il le jouait pendant cinq minutes et les gens dansaient, dansaient. [...] Ça chantait avec un seul micro et des baffles horribles, le piano était désaccordé, le son était pourri mais c'était spectaculaire. Il faisait une fusion mais super rudimentaire. Parce qu'aujourd'hui ceux qui font de la fusion, ils montent sur scène avec les trois tambours [de candombe]. Mais à cette époque, il y avait une seule tumbadora $a^{14}$, une cloche et une ou deux trompettes, pas plus ${ }^{15}$.

A l'époque Pedro Ferreira se produit dans ce qu'on appelle les bailes de la raza [«bals de la race »], des soirées dansantes majoritairement fréquentées par les AfroUruguayens. Il y joue à la fois des reprises et les chansons qu'il a écrites pour les spectacles de carnaval.

Dans les registres d'AGADU, on trouve la liste de soixante-seize compositions de Pedro Ferreira, classées par genre musical. Dix-sept candombes, sept tangos et milongas, cinq chansons de genres musicaux européens (valse, marche, scottish, jota, suite), cinq de genres brésiliens (baion et choro), une chanson enregistrée sous l'appellation « carnaval » et enfin quarante et une chansons de genres cubains (bolero, son, guaracha, conga, rumba, canción et afro). La musique cubaine représente donc plus de $50 \%$ du répertoire enregistré par Pedro Ferreira. Parmi ces chansons, six d'entre elles interpellent par leur connotation religieuse :

- Africano va a la guerra [Africain va à la guerre], 1954, avec texte et partition. Les paroles évoquent "Shango », « Ogun » et une danse « yoruba ».

- Canto a Ogun [Chant pour Ogun], sans date, avec texte et partition. Les paroles mentionnent des personnages : « Ogun », « Yemaya », « Adyinacu », « Teyú » et « Logozé ».

- Canto Toruba [Chant Toruba], 1958, avec texte et partition. Les paroles parlent d'un « royaume Toruba » de «Shango », de « Olorúm », de «Echú », et « Yfá ».

- Invocación a Ecue [Invocation à Ecue], 1959, sans texte ni partition. Le choeur Afrogama chante ce thème dont les paroles mentionnent « lemanjá » et «Shango ».

- Negro Lucumír ${ }^{16}$ [Noir Lucumi], 1954, avec texte et partition. Le texte indique que cette chanson est un « lamento esclavo », une lamentation d'esclave qui évoque la privation de liberté et la nostalgie de l'Afrique, de la jungle et des danses de la tribu.

- Santo va abla (sic) [Le saint va parler], sans date, avec texte et partition. Les parolent parlent d'un «bongo », de "Yemaya » et de « Shango ».

Ces thèmes, enregistrés sous l'appellation afro, afro-cubano ou canción, ont été composés pour Fantasía Negra, la Société de Noirs et de Lubolos que dirige à l'époque

13 Asociación General de los Autores del Uruguay.

14 La tumbadora, appelée aussi tumba ou conga, est un membranophone à une peau d'origine afro-cubaine, qu'on joue souvent par deux et qui est aujourd'hui utilisée dans toutes les musiques dites latines.

15 Entretien du 27 février 2012.

16 S'il existe des enregistrements de certaines chansons de Pedro Ferreira, en revanche il n'y a aucune trace sonore de ces chansons à connotation religieuse, sauf celles qui ont été déposées aux archives d'AGADU avec partition. 
Pedro Ferreira. En effet, dans les années 1950, la dernière partie des spectacles des Sociétés de Noirs et de Lubolos était appelée «Canción ou "Afro" " et se composait « de chants afro-cubains» (de Carvalho Neto 1967: 35). Une séquence que l'on retrouve aujourd'hui dans les spectacles sous la forme d'un «tableau afro ", évoquant l'Afrique ou les premiers temps de l'esclavage, notamment à travers la « danse d'un sorcier africain ou l'évocation d'une ancienne danse africaine» (Ferreira 1997 : 46), tableau qui sera accompagné par un thème sur un rythme appelé afro, caractérisé par une métrique ternaire et joué avec les deux mains ${ }^{17}$.

Les titres ou les paroles de ces six chansons à connotation religieuse sont remplis de références aux orishas. Les plus mentionnés sont Shangó, divinité de la virilité, du tonnerre et du tambour, Iemanjá, divinité des océans, et Ogun, divinité du feu, de la guerre et patron des forgerons. On retrouve également « Ecue », soit Ekué, nom à la fois du tambour sacré et de la voix de celui-ci dans le culte abakuá à Cuba. Dans le chant Canto Toruba sont mentionnés Olorum, dieu suprême dans les religions des orishas, et Ifá, nom renvoyant au système de divination afro-cubain Regla de Ifá, parfois confondu avec l'orisha Orunmilá, divinité de la divination, du destin, de la sagesse. Apparaît également le nom « Echú », soit Exu, nom de l'orisha messager entre les hommes et les divinités, central dans le candomblé afro-brésilien mais que l'on retrouve aussi à Cuba. De plus on trouve l'ethnonyme Yoruba et celui de Lucumí, qui était utilisé comme synonyme de Yoruba à $\mathrm{Cuba}^{18}$.

Or d'après les témoignages que j'ai pu recueillir, notamment auprès de son fils, Pedro Ferreira n'était pas initié aux religiones afro. Pourtant une partie non négligeable de son répertoire démontre une connaissance ou au moins un usage répété des noms des orishas ou de termes liés aux religions afro-cubaines, et ce, au milieu des années 1950, soit quelques années avant que l'umbanda et le batuque afro-brésiliens ne commencent leur implantation en Uruguay. Tout porte ainsi à croire que c'est bien en écoutant et en jouant de la musique cubaine qu'il s'est familiarisé avec ces éléments religieux. Car depuis les années 1920, à Cuba, sous l'impulsion du mouvement afro-cubaniste, les religions afro-cubaines sont évoquées dans le champ de la musique populaire, à travers des références aux divinités, l'usage de paroles rituelles ou du tambour bongó ${ }^{19}$. Cette appropriation est le fait de musiciens afro-cubains qui circulent entre les cérémonies religieuses et la scène musicale de l'île (Moore 2002), même si la plupart d'entre eux s'en

17 Le rythme de candombe est quant à lui construit sur une division binaire du temps et joué avec main et baguette.

18 Le terme lucumí désigne aussi la langue liturgique d'Ocha-Ifá ou le système cultuel lui-même qui réunit la Regla de Ocha ou Santería et la Regla de Ifá (Argyriadis 1999).

19 Le bongó est un membranophone composé de deux petits tambours, le plus gros nommé hembra (femelle) et le plus petit macho (mâle). D'abord utilisé par les populations d'origines africaines, notamment dans les cultes abakuá, le bongó s'est rapidement imposé dans différents répertoires, d'abord le son, au début du $20^{e}$ siècle, jusqu'au latin-jazz et à la salsa. 
inspirent sans vraiment connaître ces cultes, ni être initiés (Argyriadis 2005) ${ }^{20}$. Pedro Ferreira semble entrer dans cette catégorie. Sans posséder une véritable connaissance liturgique, il utilise les noms des divinités pour évoquer l'Afrique et ses « esprits ». Ces éléments semblent s'inscrire dans une autre dimension du «modèle de la négritude » venu des Caraïbes qui domine le marché culturel à l'époque, caractérisé notamment par la circulation d'images des «mulâtres et noirs caribéens exposant leur altérité de manière explicitement sensuelle et rythmique » (Avila Domínguez et al. 2011 : 16). Ce modèle de la négritude a une résonance particulière pour les artistes afro-uruguayens de candombe, comme en témoigne cet extrait d'un témoignage de Martha Gularte, considérée comme l'une des premières Vedettes ${ }^{21}$ du Carnaval de Montevideo, publié dans un journal national en 1950 :

« Mon arrière-grand-mère était une Africaine pure ... J'ai ce sang-là qui court dans mes veines, qui me pousse à danser de cette manière barbare et profonde, qui m'épuise, me laisse anéantie et défaite. Quand j'entends la rumeur du 'bongo', quand j'entends les sons des tambours africains, j'entre d'abord dans une langueur et une frénésie que je ne comprends pas. Ma taille devient du caoutchouc, j'ondule mon ventre, mes hanches, et je sens mes membres désarticulés... c'est comme si le saint 'Changoo' me possédait [sic] » (Acción du 6 mars 1950, cité dans Andrews 2001 : 115).

La mention du tambour bongó et de Shangó, divinité de la foudre et du tambour, témoigne de l'influence qu'ont déjà sur le milieu du candombe les productions culturelles cubaines. Ainsi, dès la fin des années 1940, l'évocation de l'africanité dans le candombe passe par l'incorporation d'éléments religieux afro-cubains déjà incorporés à la musique cubaine, sans pour autant qu'il existe à l'époque en Uruguay une connaissance pratique de ces cultes. A cette sensualité noire s'ajoute une relation à l'Afrique, à ses esprits, aux ancêtres et aux tambours, doublée d'une religiosité plus poétique et source d'imaginaires que véritablement afférée à des pratiques religieuses. De même que le passage des danses afro-américaines par le Paris Nègre va leur donner une légitimité dans leur pays d'origine, cette appropriation de la culture afro-cubaine en pleine reconnaissance va donner de la valeur à l'africanité du candombe, historiquement méprisé en Uruguay, et pose les jalons d'une culture afro-latino-américaine dépassant les identifications nationales.

\section{La rencontre du candombe de Chabela Ramírez avec les orishas}

Née en 1958 dans le quartier Palermo, tout comme Pedro Ferreira, Chabela Ramírez apprend le piano et commence à chanter dès son adolescence. Lorsqu'elle atteint la majorité à 21 ans, elle brave l'interdiction de son père et commence à se produire au

20 Deux femmes afro-cubaines en seront les visages les plus connus : Merceditas Valdés, surnommée la Pequeña Aché (La petite Aché), qui donne ses lettres de noblesse au « folklore afro-cubain » en interprétant des liturgies yorubas à la radio (Leymarie 1997 : 144), Celia Cruz et la Sonora Matancera, avec la compilation Tributo a los orishas, connue aussi sous le titre Homenaje a los santos, qui propose une anthologie de musiques de santería réarrangées dans des versions pour orchestre cubain.

$21 \mathrm{La}$ «Vedette » est la figure qui s’impose dans les défilés à partir des années 1950, fortement inspirée des danseuses de cabaret et du Carnaval de Rio de Janeiro. Elle danse, portant de grandes coiffes de plumes, perchée sur de très hauts talons avec des costumes découvrant ses formes, juste devant le groupe de tambours. 
Carnaval de Montevideo, où elle chantera dans les plus prestigieuses Sociétés de Noirs et de Lubolos, jusqu'à ce qu'elle décide, en 1991, de se mettre en grève, dénonçant l'espace subalterne réservé aux femmes et le manque de contenu des spectacles (Ferreira 2003a : 247). Cette rupture avec le milieu carnavalesque intervient dans une période particulière de sa vie. Après être «tombée amoureuse des orishas », elle commence à fréquenter à partir de 1989 un temple de batuque où elle est initiée. La pratique religieuse prend dès lors une place fondamentale dans sa pensée esthétique et politique, lui fournissant un matériau narratif, gestuel et musical dans lequel puiser pour « resignifier » et « resacraliser » le candombe ${ }^{22}$.

Mais lorsque Chabela revendique, au début des années 1990, la filiation du candombe avec le système mythologique des orishas, les rejets sont parfois très violents. Les religiones afro, bien que présentes sur le territoire depuis la fin des années 1950, sont encore méconnues et stigmatisées (Pi Hugarte 1998) dans le pays le plus laïc d'Amérique latine (Guigou 2003). Dans le milieu du candombe et le milieu militant noir, certains «traitent même Chabela de fondamentaliste », d'après sa sœur Beatríz Ramírez $z^{23}$. De l'autre côté, l'hostilité des instances religieuses à voir évoquer les orishas au sein des espaces carnavalesques de pratique du candombe est grande. Mais au fil des années, la volonté de Chabela de connecter religions afro et candombe, que Luis Ferreira qualifie de « voix discordante » au début des années 2000 (2003a : 248), va devenir une voie centrale du candombe contemporain. Même s'il reste toujours du côté des artistes de candombe et des instances religieuses des réticences à opérer cette articulation, on peut dire qu'aujourd'hui la pensée musicale de Chabela Ramírez s'épanouit dans un terreau fertile où le candombe réafricanisé et les religiones afro convergent dans la construction de l'afrodescendance en Uruguay.

Après son initiation au batuque et son boycott des spectacles de Carnaval, Chabela Ramírez va se produire dans d'autres espaces. C'est à partir de 1995, avec le groupe Afrogama, qu'elle fonde au sein de l'organisation politique noire Mundo Afro ${ }^{24}$, que Chabela Ramírez va engager ce processus de resémantisation du candombe par le religieux. Pendant douze ans, Afrogama va participer activement aux actions de Mundo Afro, puis en 2007, le groupe quitte l'organisation pour fonctionner comme une association indépendante. A la fois chœur et groupe de danse, Afrogama est composé entièrement de femmes, certaines catégorisées comme noires et d'autres comme blanches, qui vivent dans des conditions socio-économiques contrastées, allant de la grande précarité à la classe moyenne. Si la majorité d'entre elles n'étaient pas initiées aux religiones afro quand j'ai commencé ma recherche en 2007, en dix ans j'ai pu voir

22 Chabela Ramírez poursuit également la dynamique de celui qu'elle présente comme son mentor, José Agapito Carrizo, écrivain et militant qui a été le premier à théoriser l'articulation du candombe avec la lutte politique et à prôner l'initiation aux religions afro (Ferreira 2003a : 164). Pour une analyse plus détaillée de la trajectoire de Chabela Ramírez, voir Biermann 2012.

23 Extrait d'un entretien daté du 4 février 2008.

24 Sur l'histoire de cette organisation créée en 1989 et son impact majeur dans la reconnaissance des inégalités raciales en Uruguay, l'élaboration d'un discours d'auto-estime noire et l'usage du candombe comme instrument politique, voir Ferreira 2003a et 2003b. Sur leur participation à la constitution d'un réseau transnational d'organisations afro-latino-américaines, voir Cunin 2006. 
un nombre croissant d'entre elles s'initier ou avoir recours aux services d'autorités religieuses de batuque ou d'umbanda.

Les femmes d'Afrogama (photo 2), sous la direction de Chabela, privilégient des versions polyphoniques de chansons de candombe, uniquement accompagnées par les tambours, choisies pour leurs thématiques traitant du droit des femmes, de l'égalité sociale et raciale, ou racontant l'histoire de l'esclavage. On trouve aussi dans leur répertoire des chansons à connotation religieuse, comme Invocación a Ekué de Pedro Ferreira, Eleguá quiere tambó, une reprise de la chanson interprétée par Celia Cruz et la Sonora Matancera, ou Candombe Candomblé, l'hymne du groupe composé par Chabela Ramírez, qui par un jeu de glissement sémantique affirme la connexion entre le genre musical afro-uruguayen et la religion afro-brésilienne ${ }^{25}$.

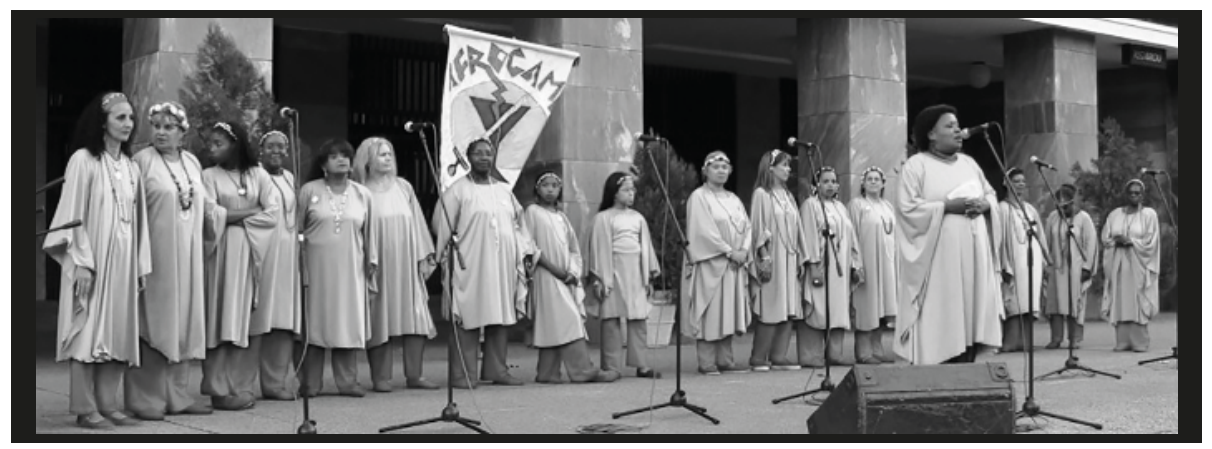

Photo 2. Le choeur Afrogama sur l'esplanade de la Mairie de Montevideo, à l'occasion de la Journée de la Femme 2009 - (C) Clara BIERMANN

A partir de 1998, le groupe Afrogama va se produire dans un espace de grande visibilité nationale, en ouverture du Défilé de Llamadas (photo 3) - le concours annuel des groupes de tambours et de danseuses. Le groupe Afrogama défile hors-concours, accompagné par les tambours de l'Ecole de Candombe de Mundo Afro, et danse des chorégraphies créées par Chabela Ramírez, représentant les orishas, par un figuralisme gestuel de leurs caractéristiques mythologiques ou de l'élément naturel dont ils sont l'incarnation ${ }^{26}$.

A partir de 2001, Chabela veut intégrer les orishas « en chair et en os » dans le défilé et fait appel à la Fédération afro-umbandiste Atabaque, fondée en 1997 par le couple Julio Kronberg et Susana Andrade, pour qu'ils viennent incarner leurs orishas dans le défilé. Julio Kronberg-Pai Julio de Omulú et Susana Andrade-Mãe Susana de Oxúm sont père et mère-de-saints d'umbanda à Montevideo et sont des militants religieux de longue date.

25 Sur les procédés utilisés par le chœur Afrogama pour « noircir » le trait musical et réafricaniser le candombe, voir Biermann 2011.

26 Pour une analyse complète des créations chorégraphiques de Chabela Ramírez représentant les orishas avec le groupe Afrogama, voir Biermann 2017. 


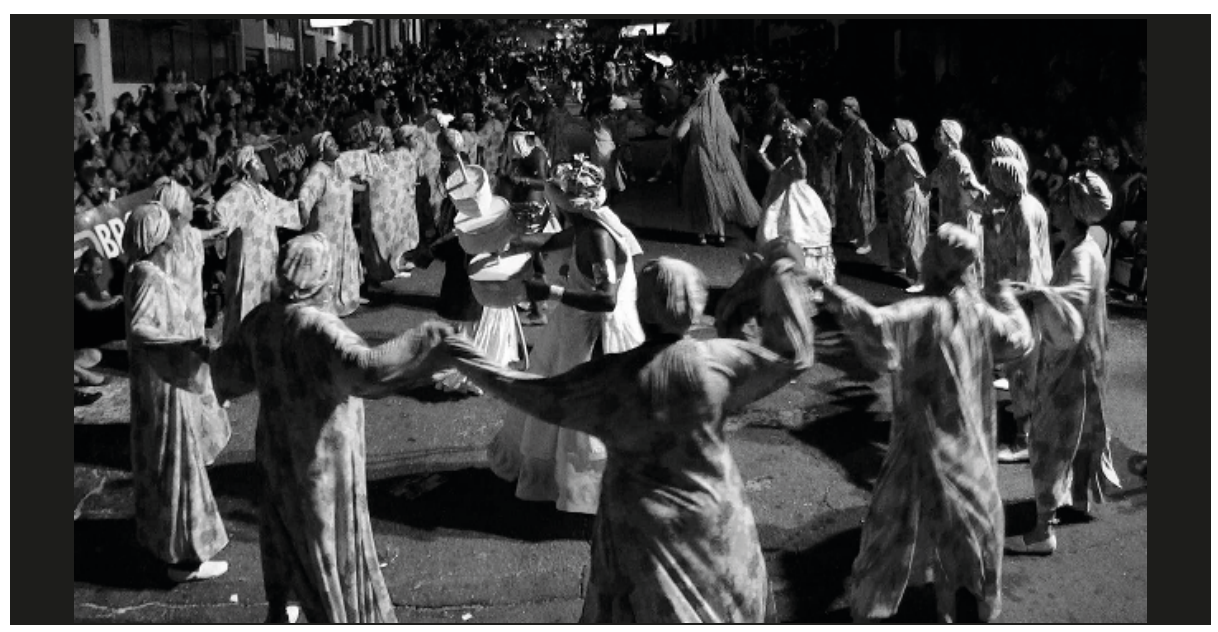

Photo 3. Le groupe Afrogama habillé en l'honneur de lemanjá, entourant les orishas au Desfile de Llamadas, 2010 - (C) Laura GANZ

A cette époque, la Fédération Atabaque est mobilisée dans la "guerre sainte » qui oppose, dans le Cône Sud, les umbandistes et l'Eglise Universelle du Royaume de Dieu, une puissante organisation néo-pentecôtiste brésilienne (Frigerio \& Oro 2005). Alors que leurs cultes sont violemment attaqués, les umbandistes cherchent à dédiaboliser les religiones afro en nouant des alliances avec des secteurs sociaux plus larges, et notamment avec l'organisation politique noire Mundo Afro (ibid.). Mais au sein de Mundo Afro, c'est en fait avec Chabela Ramírez et son groupe Afrogama que s'opère cette alliance. Mãe Susana de Oxúm ${ }^{27}$, qui pense les religiones afro comme la racine de la culture noire, et Chabela Ramírez, qui veut resacraliser le candombe, vont étroitement collaborer et jouer un rôle de médiatrices entre le milieu religieux et le milieu musical. Leur alliance incarne la convergence qui s'opère entre la légitimation des religions dans le Cône Sud, qui tient « à leur degré d'insertion - en raison de leurs origines noires dans les constructions narratives historiques dominantes de chaque nation ou chaque région » (Frigerio \& Oro 2005), et le candombe, qui va opérer comme une interface et un ancrage, en territorialisant les religiones afro au sein de "l'intimité culturelle» uruguayenne (Herzfeld [1997] 2007). Depuis 2009, Chabela Ramírez poursuit cette incorporation des orishas dans le Desfile de Llamadas avec le groupe dirigé par son fils Diego Paredes, qui participe chaque année au concours et qui est passé de la dixhuitième place au classement général à la deuxième en 2018, focalisant d'autant plus l'attention nationale sur le groupe Afrogama et ses orishas.

27 Susana Andrade-Mãe Susana de Oxúm milite inlassablement pour la reconnaissance des religiones afro à l'échelle nationale. Figure médiatique de l'umbanda, elle a été élue en 2014 députée suppléante du parti de gauche Frente Amplio à l'Assemblée, devenant la première « sorcière ", selon le terme qu'elle a employé le jour de l'élection, à accéder à la plus haute sphère décisionnelle du pays. 


\section{Resacraliser le candombe ? Procédés musicaux et poétiques}

Dans de précédentes publications, j’ai analysé la manière dont Chabela Ramírez avec le groupe Afrogama «noircit» le trait musical pour réafricaniser le candombe chanté (Biermann 2011) à partir de procédés musicaux qui fonctionnent en miroir avec ses créations chorégraphiques représentant les orishas (Biermann 2017). Je propose ici de limiter l'échelle d'analyse à une chanson, extraite du premier disque soliste de Chabela Ramírez, qui s'inscrit dans la continuité de son travail musical avec Afrogama depuis une vingtaine d'années. Intitulé De tambores y de amores (photo 4), ce disque est sorti en 2016 en Uruguay, à l'issue d'une collaboration franco-uruguayenne dont j'ai été la coordinatrice, ce qui m'a amenée à en suivre toutes les étapes de réalisation ${ }^{28}$.

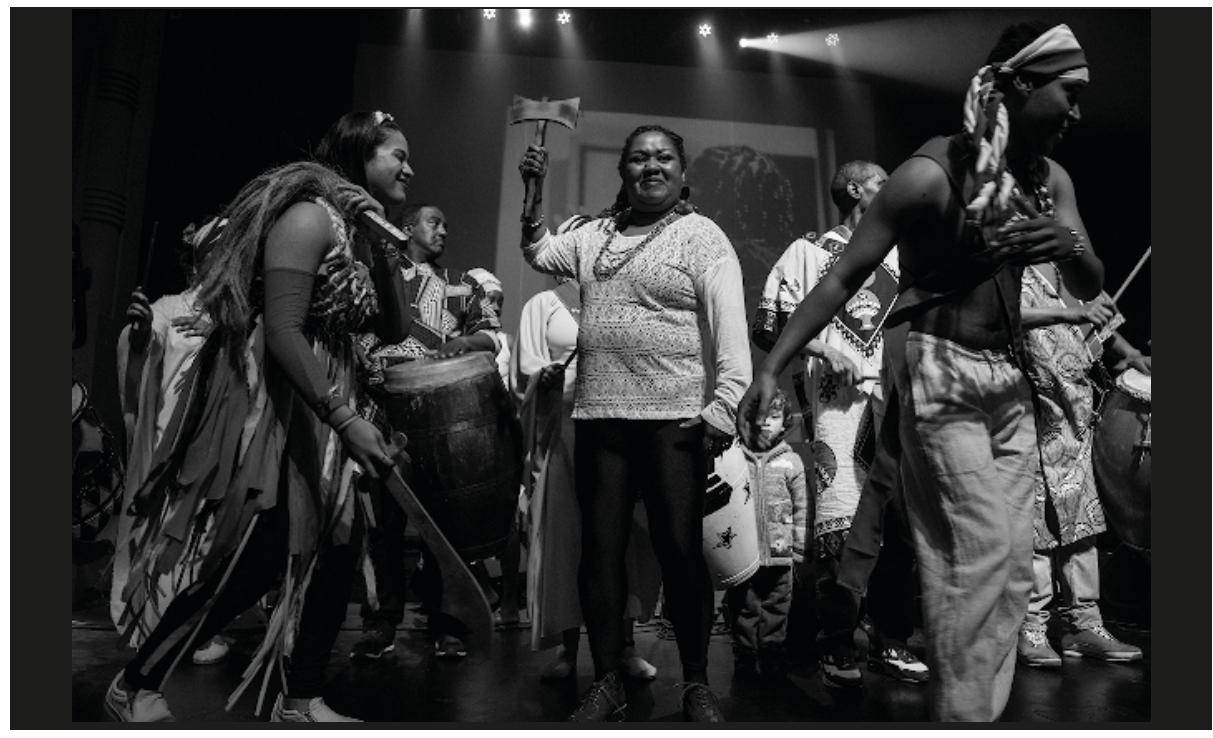

Photo 4. Chabela Ramírez tenant la double hache de Shangó, accompagnée de danseurs et danseuses représentant leur orisha. Concert de présentation du disque De tambores y de amores, Montevideo, 28 août 2016 - (C) Mauro MARTELLA

Cet objet discographique a représenté pour Chabela Ramírez l'occasion de revenir sur une vie de musique et d'engagement. Chaque chanson a été choisie avec soin, pour évoquer la place de la femme noire, le racisme, l'amour du candombe et du tambour, et bien sûr les religiones afro. Sur un total de douze titres, De tambores y de amores comprend trois chansons à connotation religieuse. Deux d'entre elles sont des chansons du répertoire d'Afrogama réarrangées avec un instrumentarium plus varié. La première, Eleguá quiere tambó, reprise de Celia Cruz et la Sonora Matancera, est interprétée avec marimba, berimbau, chœur féminin et tambours de candombe, et Candombe Candomblé, avec basse, guitare, ensemble de cuivres, chœur féminin et tambours de candombe. Je m'intéresserai en détail à la troisième, intitulée Alabanza a los orixás (Louange aux

28 Pour une analyse complète du disque De tambores y de amores, voir Cardoso 2017. 
orishas), interprétée avec voix, chœur féminin, tambours de candombe et berimbau ${ }^{29}$. Alabanza a los orixás est un popurri (du français pot-pourri), un medley réunissant trois chansons que nous allons traiter ici une par une pour la clarté de l'analyse. La première partie du popurri est une composition de Chabela Ramírez écrite en 2011 pour Afrogama. Construite sur une structure responsoriale et d'abord destinée à être chantée par le groupe de danse lors du défilé de Llamadas, elle est d'abord appelée Canto a los orixás (Chant pour les orishas), puis elle prend le nom de Rezo (Prière) et connaît plusieurs versions successives ${ }^{30}$.

\section{Alabanza a los orixás}

Exergue poétique: Cuantos cantos desde el cielo De espiritus y ancestros buenos Alupo
Combien de chants depuis le ciel D'esprits et ancêtres bienfaiteurs Alupo

Première partie de la chanson : Rezo, de Chabela Ramírez

Bará pido agô y ashé (bis par le choeur)

Bará dice que pasen (bis)

Ogun pido agô y ashé (bis)

Ogun dice que marchen (bis)

Oia pido agô y ashé (bis)

Oia dice que canten (bis)

Shangó pido agô y ashé (bis)

Shangó dice que danzen (bis)

Bará con Ogun, Shangó con Oia

Llegan orishas a candombear

Epao Babá, Oshun lemanjá

Llegan orishas a candombear

Ansina esta de fiesta

Te invita a candombear

Renace mi Palermo

Con su energía / Y su alegría
Bará, je demande agô et ashée ${ }^{31}$

Bará dit : passez

Ogun, je demande agô et ashé

Ogun dit : marchez

Oia, je demande agô et ashé

Oia dit : chantez

Shangó, je demande agô et ashé

Shangó dit : dansez

Bará avec Ogun, Shangó avec Oia

Les orishas arrivent pour le candombe

Epao Babá, Oshun, lemanjá

Les orishas arrivent pour le candombe

Ansina est en fête

Il t'invite pour le candombe

Mon Palermo [le quartier] renaît

Avec son énergie / Et son allégresse

Chaque vers commence par la mention d'un orisha à qui sont demandés agô et ashé, soit " permission et pouvoir» (traduction de Chabela), avant les actions du groupe. Chabela commence par demander à Bará32, qui préside au destin des hommes et qui est invoqué avant de réaliser toute chose. Permission et pouvoir sont ensuite demandés à Ogun, puis à Oia, divinité féminine des tempêtes, et à Shangó. Par ces paroles, les actions effectuées par le groupe, passer, marcher, chanter et danser, qui décrivent le déroulement d'une sortie de tambours de candombe, sont sanctifiées par les divinités et

29 Pour écouter la chanson Alabanza a los orixás, consultation libre : < https://archives.crem-cnrs.fr/ archives/items/CNRSMH_E_2018_009_001_06/>

30 Avant la sortie du disque, Chabela Ramírez a interprété sa chanson Rezo lors d'un cycle de concerts consacrés au candombe organisé par AGADU. Voir : <https://www.youtube.com/watch? $\mathrm{v}=$ bvE0o6E6-g>

31 Le terme yoruba agô veut dire permission. Le terme ashé désigne la force sacrée inscrite dans les objets et les personnes initiées, une force qui est transmissible.

32 Dans le batuque, Bará remplace Exu en tant que messager entre les hommes et les divinités. Voir Frigerio, $<$ http://www.ird.fr/relitrans/spip.php?article319> 
prennent une dimension rituelle. Arrivent ensuite les autres divinités, « en personne ». Oshalá, orisha créateur de la terre et de l'être humain et maître des pensées, est invoqué par la salutation rituelle «Epao Babá ». Puis sont aussi appelées Oshun, orisha de la féminité, la maternité et l'amour charnel, et Iemanjá, divinité des océans et mère de tous les orishas, pour participer à la sortie de tambours. Évoquant le principe de la descente des divinités dans le corps des initiés et dans le monde des hommes, l'incorporation des orishas se fait ici à travers un corps collectif qui marche et danse au son des tambours de candombe, dans le quartier Palermo.

La deuxième partie de la chanson change de registre et passe à une célébration du quartier Palermo et des maisons de la rue Ansina - nom d'un ensemble de maisons détruit pendant la dictature, considéré comme l'un des foyers historiques du candombe et où vivaient la majorité des membres de Fantasía Negra, la Société de Noirs et de Lubolos dirigée par Pedro Ferreira dans les années 1950. Le couplet s'inscrit dans une tradition d'hommage à ce lieu disparu et au quartier, que l'on retrouve dans de nombreuses chansons et qui s'inscrit dans une topographie culturelle de la ville de Montevideo. D'un point de vue musical, l'orchestration de la chanson se rapproche de l'esthétique déjà privilégiée par le groupe Afrogama : une voix soliste et un chœur féminin uniquement accompagnés par les tambours de candombe et par un berimbau, l'arc musical à résonateur à calebasse utilisé dans la capoeira brésilienne, qui marque certains premiers temps du cycle rythmique avec son hochet et une frappe sur la corde dont le timbre caractéristique est riche en harmoniques. Notons bien cependant que, si les orishas sont invoqués, la composition de Chabela ne reprend pas les formules mélodico-rythmiques rituelles de chaque orisha, et il ne s'agit en aucun cas d'une adaptation des répertoires liturgiques.

De longs secouements de hochet du berimbau et des roulements de tambours annoncent la transition vers la seconde chanson du popurri, Canto a Ogun, qui fait partie des compositions de Pedro Ferreira archivées à AGADU. Quelques jours avant mon départ de Montevideo en mars 2010, je montre à Chabela la liste des chansons de Pedro Ferreira que je venais de récupérer à AGADU. Très émue, elle repère toute de suite cette chanson dont elle se souvenait, parce que, me dit-elle, on la chantait dans les fêtes du quartier. Sans regarder la partition, elle fredonne l'air et se réjouit d'avoir retrouvé les paroles exactes, tout en essayant de se remémorer le rythme exact de son accompagnement rythmique. En 2013, lors de l'enregistrement du disque, elle décide de l'intégrer à son popurri religieux, réactivant ainsi les « premiers » orishas du candombe, ceux de Pedro Ferreira. 
Canto a Ogun - Paroles et musique : Pedro Ferreira

O lemanjá aé

Ayinacu se enojo aé

Exú dijo no puede ser

Ogun yo te quiero ver

Te quiero ver

Sale a brillar para ti

Con tu rayos alumbrar

Esta penumbra

Solo tu puede dar

Vida y calor Ogun

Mis brazos ya están cansados

$Y$ esperar no quieren (bis)

Tambao yo pedi a Ifa

Tu me va' decir

Ogun donde está (ter)
Oh lemanjá aé

Ayinacu s'est fâché aé

Exu a dit : ce n'est pas possible

Ogun, je veux te voir

Je veux te voir

Il vient briller pour toi

Avec tes rayons éclairer

Cette pénombre

Seul toi tu peux donner

De la vie et de la chaleur, Ogun

Mes bras sont déjà fatigués

Et ils ne veulent pas attendre

Tambao, j’ai demandé à lfá

Toi, tu vas me dire

Où est Ogun

Evoquant les orishas Iemanjá, Ogun, mais également un certain « Teyú » (tel qu'écrit dans les archives d'AGADU), que Chabela a interprété comme étant Exu, messager entre les hommes et les divinités (appelé aussi Bará dans le batuque et Eleguá à Cuba), la chanson demeure auréolée d'un certain mystère, même pour Chabela qui me confessait ne pas savoir qui était «Ayinacu». Sans pouvoir établir de comparaison, faute d'enregistrement original, on peut dire que l'interprétation de Chabela est épurée : la voix est nue, uniquement accompagnée par les tambours, comme c'est le cas dans les cérémonies religieuses. La chanson est en tonalité mineure, comme la plupart des chansons évoquant l'Afrique à cette époque dans les musiques latino-américaines. Sur une rythmique superposant des motifs binaires et ternaires, évoquant la rythmique afro et jouée sur les tambours avec les deux mains, Chabela chante seule, rejointe par une deuxième voix qu'elle a elle-même enregistrée.

La troisième partie du medley est une chanson intitulée El Canto a Yanaina ${ }^{33}$, qui fait partie du répertoire d'Afrogama depuis de nombreuses années et avec laquelle Chabela entretient une relation particulière. Composée par Rodolfo Morandi (musique) et Miguel Ángel Herrera (paroles), cette chanson est la première que Chabela interprète sur la scène du Carnaval de Montevideo en 1980. Pendant les répétitions, Miguel Ángel Herrera, dont plusieurs sources affirment qu'il pratiquait les religiones afro, lui dit: « Tu dois y croire [...]. Cette chanson doit être chantée par une fille de Shangó », sans que Chabela comprenne de quoi il s'agit. Le soir de sa prestation, raconte-t-elle, les gens lui disent qu'elle a chanté comme si elle était en train de regarder Shangó. " Difficile de penser que c'était une coïncidence », ajoute Chabela, qui sera, une dizaine d'années plus tard, initiée à l'orisha Shangó dans le batuque.

33 Pour les orishas, on ne parle pas d'âges mais de passages. Yanaina est le nom d'un passage enfant de Iemanjá. 


\begin{tabular}{|c|c|}
\hline Vamos todos al canto de Yanaina & Allons tous au chant de Yanaina \\
\hline Vamos todos & Allons-y tous \\
\hline Que lemanjá nos va a esperar & Parce que lemanjá nous attend \\
\hline Traigan flores & Apportez des fleurs \\
\hline Y alguna vela encendida & Et une chandelle allumée \\
\hline Yagua santa que nos regaló lemanjá & Et de l'eau bénite que nous a offerte lemanjá \\
\hline Mi Pai Shangó & Mon Pai Shangó \\
\hline Me protege & Me protège \\
\hline Y es el guía de mi vida & Et c'est le guide de ma vie \\
\hline Por eso yo & C'est pour ça \\
\hline Voy contenta al canto de Yanaina & Que je vais heureuse au chant de Yanaina \\
\hline Vamos todos al canto de Yanaina & Allons tous au chant de Yanaina \\
\hline Vamos todos & Allons-y tous \\
\hline Que lemanjá nos va a esperar & Parce que lemanjá nous attend \\
\hline Traigan flores & Apportez des fleurs \\
\hline Y alguna vela encendida & Et une chandelle allumée \\
\hline Y agua santa que nos regaló lemanjá & Et de l'eau bénite que nous a offerte lemanjá \\
\hline Nos vamos ya & Allons-y maintenant \\
\hline Traigan flores & Apportez des fleurs \\
\hline Y alguna vela encendida & Et une chandelle allumée \\
\hline
\end{tabular}

Entraînante et lumineuse, construite sur des harmonisations majeures, la chanson $E l$ Canto a Yanaina parle de Iemanjá et des offrandes rituelles qui lui sont destinées le jour de sa fête. Elle est entonnée sur rythme de candombe rapide qui se prolonge en une partie consacrée au jeu des tambours de candombe, accompagnés par le berimbau, avec des jeux d'accélération et d'intensité.

Alabanza a los orixás met ainsi en cohérence la composition de Chabela composée en 2011, celle de Pedro Ferreira au milieu des années 1950 et celle de Morandi et Herrera écrite en 1980. Le popurri construit un objet musical et narratif qui fait s'entrelacer trois temporalités créatives dans lesquelles les orishas sont présents dans le candombe. Pour Chabela, la présence des orishas dans les chansons de Pedro Ferreira est une preuve que les divinités africaines étaient déjà présentes avant l'implantation des religions afrobrésiliennes en Uruguay, et vient affirmer l'existence d'une religiosité « ancestrale » dans le candombe, argument fondamental de sa pensée artistique et politique. Il reste une enquête à mener sur la réception de ce disque dans le milieu musical et plus particulièrement sur la manière dont les auditeurs perçoivent les différents registres de signification mobilisés par Chabela Ramírez. Mais on peut déjà avancer que, même si ces auditeurs ne maîtrisent pas forcément toutes les références, les choix poétiques et musicaux de Chabela Ramírez sont intelligibles et ont un effet pratique. L'usage de la polyphonie, le jeu d'entrelacement voix et tambours, l'utilisation du berimbau, la rythmique afro en $6 / 8$ jouée avec les deux mains, renvoient aux musiques religieuses et à des imaginaires historiques et culturels de l'africanité latino-américaine. Ces inflexions sont d'autant plus identifiables qu'elles contrastent avec le style des candombes joués 
au Carnaval de Montevideo, de même qu'avec celui des artistes catégorisés comme Blancs, lié à l'esthétique de la chanson engagée des années 1970, où le candombe est évoqué dans le jeu rythmique de la guitare et dans les paroles des chansons (Picún 2016).

On peut considérer que Chabela Ramírez procède par appropriation, ce que DenisConstant Martin définit comme «l'adoption [...] de traits musicaux, de genres, de styles [...] considérés comme appartenant à des univers musicaux différents de celui de 1'“emprunteur" "(2014: 49). Mais du point de vue de Chabela, ces inflexions esthétiques et religieuses s'appuient sur des éléments qui ne sont pas considérés comme exogènes. Les orishas sont évoqués comme ces divinités partagées par la diaspora noire des Amériques venant sanctifier la pratique du candombe dans le quartier Palermo de Montevideo. Les imaginaires afrodescendants s'articulent à l'affirmation d'une tradition musicale locale, sans aucune forme de contradiction. En articulant différentes temporalités, cette chanson vient ainsi affirmer une ancestralité reconstruite, où l'Afrique est un espace autant qu'un temps des origines.

\section{Conclusion}

La première phase d'incorporation des orishas dans le candombe date des années 1950 et résulte de l'appropriation de l'esthétique des musiques afro-cubaines, largement diffusées à l'échelle internationale. Celles-ci représentent alors un modèle de la négritude, qui va avoir un très fort impact sur les musiciens de candombe qui sont en négociation constante avec leurs origines africaines tout en cherchant à construire leur légitimité culturelle dans le marché musical local. La seconde étape, à partir des années 1990, s'inscrit quant à elle dans une convergence idéologique et esthétique entre les religiones afro et le candombe, dont Chabela Ramírez a été la pionnière.

Dans les années 1950, la démarche de Pedro Ferreira n'est pas militante. Les signifiants poétiques et sonores empruntés à la musique cubaine sont fragmentés et ils sont plutôt utilisés pour évoquer l'Afrique que pour parler de la pratique de ces religions afrocubaines. Cependant les compositions de Pedro Ferreira, accompagnant des tableaux théâtraux et dansés dans les spectacles au Carnaval de Montevideo, performent une afro-uruguayennité qui puise dans des référents circulant à l'échelle globale, tout en entrant en résonance avec les enjeux de l'identité locale. Le candombe de Chabela Ramírez a une dimension plus prosélyte et veut affirmer une ancestralité africaine par le biais d'une mise en présence de ces divinités partagées par les Afrodescendants en Amérique latine.

Même si, pour Chabela, l'objectif est de resacraliser le candombe, le cas présenté ici ne relève pas pour autant de la sacralisation d'une musique profane. Un tel processus nécessiterait des instances de légitimation et de ratification des autorités religieuses afro-umbandistes elles-mêmes. Or les tambours de candombe ne sont pas utilisés dans les maisons de culte pour invoquer les orishas, et les tambours sacrés de l'umbanda et du batuque ne sont (pas encore) joués ni dans le Desfile de Llamadas ni sur la scène du Carnaval de Montevideo. Malgré ces inflexions religieuses et même s’il se voit enrichi de 
nouvelles significations, le candombe n'a pas changé de registre d'existence. Il subsiste en ce sens une imperméabilité entre les espaces religieux et les espaces de pratique du candombe. En revanche la réafricanisation est articulée à l'appropriation d'une forme de sacralité conférée par les divinités yorubas. Le candombe, travaillé par les artistes, se voit doté de « pouvoirs enchanteurs » (Sullivan 1997) autant qu'il retrouve et approfondit son africanité. Ces circulations et appropriations dessinent les réseaux des « connexions diasporiques » qui participent à l'élaboration d'un " patrimoine culturel "afro" transnational » (Capone 2011), dans un Atlantique noir (Gilroy [1993] 2003) qui doit être pensé avec l'Amérique latine, et dans lequel les artistes afro-américains définissent génération après génération une afrodescendance politique sensible.

\section{Références citées}

Agier, Michel, 2000. Anthropologie du carnaval : la ville, la fête et l'Afrique à Bahia. Paris : IRD /Marseille : Editions Parenthèses.

Aharonián, Coriún, 2010. Músicas populares del Uruguay. Montevideo : Universidad de la República.

Alfaro, Milita, 1991. Carnaval. Una historia de Montevideo desde el punto de vista de la fiesta. Primera Parte. Montevideo : Trilce.

Andrews, George R., 2011. Negros en la nación blanca : historia de los afrouruguayos, 18302010. Montevideo : Linardo y Risso.

Argyriadis Kali, 1999. «Une religión vivante. Continuité et complémentarité des pratiques cultuelles havanaises ", L'Homme 151, pp. 21-46.

Argyriadis Kali, 2005. «Les batá deux fois sacrés : la construction de la tradition musicale et chorégraphique afro-cubaine », Civilisations 53 (1), pp. 45-74.

Argyriadis, Kali \& Stefania Capone, 2011. La religion des orishas. Un champ social transnational en pleine recomposition. Paris : Hermann.

Ávila Domínguez Freddy, Ricardo Pérez Montfort \& Christian Rinaudo (éds), 2011. Circulaciones culturales. Lo afrocaribeño entre Cartagena, Veracruz y La Habana. Mexico : Publicaciones de la Casa Chata.

Ayestaran, Lauro, 1953. Las músicas primitivas en el Uruguay. Montevideo : Sodre.

Bastide, Roger, [1967] 1996. Les Amériques noires. Paris : L'Harmattan.

Bastide, Roger, [1958] 2000. Le candomblé de Bahia. Paris : Plon.

Bernand, Carmen, 2013. " "Musiques métisses", musiques criollas. Sons, gestes et paroles en Amérique Hispanique », L'Homme 207-208, pp. 193-214.

Biermann, Clara, 2011. « Jeux de couleurs dans le candombe afro-uruguayen », Volume! 8 (1), pp. 131-147.

Biermann, Clara, 2012. « La voie de Chabela. Trajectoire d'une figure du candombe afrouruguayen », in Emmanuelle Olivier (éd.), Musique au monde. La tradition au prisme de la création, pp. 47-66. Paris : Delatour.

Biermann, Clara, 2015. « Les visages du candombe. Pratiques, création et savoir-faire chez les musiciens et les danseurs afro-uruguayens ", thèse de doctorat en ethnomusicologie, Université Paris Nanterre, 496 p.

Biermann, Clara, 2017. « Faire corps avec les orishas. Création chorégraphique et construction d'afrodescendance en Uruguay », in Emmanuelle Olivier \& Sarah Andrieu (éds), Création artistique et imaginaires de la globalisation, pp. 329-349. Paris : Hermann. 
Capone, Stefania, 1999. La quête de l'Afrique dans le candomblé : pouvoir et tradition au Brésil. Paris : Karthala.

Capone, Stefania, 2004. "A propos des notions de globalisation et de transnationalisation », Civilisations 51(1-2), pp. 9-22.

Capone, Stefania, 2005a. «Repenser les "Amériques Noires" : nouvelles perspectives de la recherche afro-américaniste I », Journal de la Société des Américanistes 91 (1), pp. 83-91.

Capone, Stefania, 2005b. Les Yorubas du Nouveau Monde : religion, ethnicité et nationalisme noir aux Etats-Unis. Paris : Karthala.

Capone, Stefania, 2011. "Connexiones "diaspóricas" : redes artísticas y contrucción de un patrimonio cultural "afro" », in Freddy Avila Dominguez, Ricardo Perez Montfort \& Christian Rinaudo (éds.), Circulaciones culturales. Lo afrocaribeño entre Cartagena, Veracruz y La Habana, pp. 217-246. Mexico : Publicaciones de la Casa Chata.

Cardoso, Ignacio, 2017. « Uruguay. De tambores y de amores, Chabela Ramírez », Cahiers d'ethnomusicologie 30, pp. 250-254.

Cunin, Elisabeth, 2006. « La "diaspora noire" est-elle latine ? Ethnicité, nation et globalisation en Colombie », Autrepart 38 (2), pp. 135-154.

de Carvalho Neto, Paulo, 1967. El Carnaval de Montevideo : folklore, historia y sociología. Seville : Universidad de Sevilla.

Décoret Ahiha, Anne, 2004. Les danses exotiques en France (1880-1940). Pantin : Centre national de la danse.

Ferreira, Luis, 1997. Los tambores del candombe. Montevideo : Colihue-Sepé.

Ferreira, Luis, 2003a. «"Mundo Afro": Uma História da Consciência Afro-Uruguaia no seu processo de emergencia ». Thèse de doctorat en anthropologie sociale, Universidade de Brasília.

Ferreira, Luis, 2003b. El Movimiento Negro en el Uruguay (1988-1998) : una versión posible. Avances en el Uruguay post-Durban. Montevideo : Ediciones Étnicas, Mundo Afro.

Frigerio, Alejandro, 1998. «El rol de la "escuela uruguaya" en la expansión de las religiones afrobrasileñas en Argentina », in Renzo Pi Hugarte (éd.), Cultos de Posesión en el Uruguay: Antropología e Historia, pp. 75-98. Montevideo : Ed. de la Banda Oriental.

Frigerio, Alejandro, 2011. «L'umbanda et le batuque dans le Cône Sud : du flux religieux transfrontalier au champ social transnational », in Argyriadis Kali \& Stefania Capone (éds), La religion des orisha : un champ social transnational en pleine recomposition, pp. 273322. Paris : Hermann.

Frigerio, Alejandro \& Ari Pedro Oro, 2005. « Guerre sainte dans le Cône sud latino-américain pentecôtistes versus umbandistes », Journal de la société des américanistes 91 (2), pp. 185218.

Gilroy, Paul, [1993] 2003. L'Atlantique noir. Modernité et double conscience. Paris : Edition Kargo.

Herzfeld, Michael, [1997] 2007. L'intimité culturelle. Poétique sociale dans l'Etat nation. Québec : Presses de l'Université Laval.

Jacotot, Sophie, 2008. « Genre et danses nouvelles en France dans l'entre-deux-guerres », Clio. Histoire, femmes et societés 27, pp. 225-240.

Guedj, Pauline, 2011. Panafricanisme, religion akan et dynamiques identitaires aux Etats-Unis. Le chemin du Sankofa. Paris : L'Harmattan.

Guigou, Nicolás, 2003. La Nación laíca : religión civil y mito-praxis en el Uruguay. Montevideo : Ediciones La Gotera. 
Halloy, Arnaud, 2005. Dans l'intimité des orixás : corps, rituel et apprentissage religieux dans une famille-de-saint de Recife, Brésil. Thèse de doctorat en anthropologie sociale (Université libre de Bruxelles) et ethnologie (EHESS, Paris).

Leymarie, Isabelle, 1997. Cuban Fire : musiques populaires d'expression cubaine. Paris : Editions Outre Mesure.

Martin, Denis-Constant, 2014. «Attention, une musique peut en cacher une autre. L'appropriation, A et $\Omega$ de la création ", Volume! 10 (2), pp. 47-67.

Moore, Robin D, 2002. Música y mestizaje. Revolución artística y cambio social en La Habana, 1920-1940. Madrid : Colibri.

Olivera Chirimini, Tomás \& Juán Ángel Varese, 2000. Los candombes de reyes. Las llamadas. Montevideo : Ed. El Galeón.

Pi Hugarte, Renzo (éd.), 1998. Los cultos de posesión en el Uruguay. Antropologia e Historia. Montevideo : Banda Oriental.

Picún, Olga, 2016. « Cambio, identidad y crítica : el candombe en el movimiento de la Música Popular Uruguaya », Cahiers d'Etudes des Cultures Ibériques et Latino-américaines 2, pp. 31-56.

Stokes, Martin, (éd.), 1994. Ethnicity, identity and music. The musical construction of place. Oxford \& New York : Berg Publishers.

Sullivan, Lawrence E., (éd.), 1997. Enchanting powers. Music in the world's religions. Cambridge : Harvard University Press.

Verger, Pierre Fátúmbí, [1954] 1995. Dieux d'Afrique : culte des Orishas et Vodouns à l'ancienne Côte des Esclaves en Afrique et à Bahia, la Baie de Tous les Saints au Brésil. Paris : Editions Revue Noire.

\section{Discographie}

Chabela Ramírez. 2016. De tambores y de amores. Montevideo : Perro Andaluz. Livret de 12 pages. CD PA6717-2.

Chabela Ramírez (chanteuse), Clara Biermann (collecteur), 2016. Alabanza a los orixás. Enregistrement sonore : consultation libre : $<$ https://archives.crem-cnrs.fr/archives/ CNRSMH_E_2018_009_001_06/>

Archives sonores du CNRS/Musée de l'Homme, gérées par le Centre de recherche en ethnomusicologie (LESC-CNRS - Université Paris Nanterre) avec le soutien du Ministère de la Culture et de la Communication. 